\title{
Vitrectomy with and without encircling band for pseudophakic retinal detachment with inferior breaks: VIPER Study Report No. 3
}

\author{
Sabine Baumgarten ${ }^{1}$ (D) Petra Schiller ${ }^{2} \cdot$ Martin Hellmich $^{2} \cdot$ Peter Walter $^{1} \cdot$ Hansjürgen Agostini $^{3} \cdot$ Bernd Junker $^{4}$. \\ Horst Helbig ${ }^{5}$. Albrecht Lommatzsch ${ }^{6} \cdot$ Babac Mazinani $^{1} \cdot$ on behalf of the VIPER Study Group
}

Received: 10 June 2018 /Revised: 31 July 2018 / Accepted: 13 August 2018 / Published online: 23 August 2018

(C) The Author(s) 2018

\begin{abstract}
Purpose To test if an encircling band improves outcomes in vitrectomy for pseudophakic retinal detachment (PRD) with inferior or with multiple (4 or more) breaks.

Methods Subgroup analysis of a prospective randomized controlled multicenter trial in patients with uncomplicated PRD assigned either to $20 \mathrm{G}$ vitrectomy plus encircling band (group E1), or $20 \mathrm{G}$ vitrectomy without any buckle (group C), or 23/25 G vitrectomy without any buckle (group E2). The primary endpoint was defined as no indication for any retina reattaching procedure during the review period of 6 months. One hundred out of 257 patients were identified with inferior breaks and 63 patients had 4 or more breaks. Results In patients with retinal breaks between 5:00 and 7:00, treatment was successful in 77.4\% (24/31, treatment arm E1) versus $57.1 \%$ (16/28, treatment arm C) $(p=0.301$, odds ratio (OR) $1.83,95 \%$ confidence interval (CI) 0.48 to 7.17$)$. In patients with multiple breaks, success rates were $68.2 \%(15 / 22$, E1) versus. $72.4 \%$ (21/29, C, $p=0.46$, OR 0.52, CI 0.08-3.65).

Conclusion Combining an encircling band with vitrectomy in patients with pseudophakic retinal detachment and inferior or multiple breaks does not significantly improve primary anatomical success in comparison to treatment with $20 \mathrm{G}$ or $23 / 25 \mathrm{G}$ vitrectomy alone.
\end{abstract}

Keywords Inferior breaks · Pseudophakic · Randomized clinical trial $\cdot$ Retinal detachment $\cdot$ Scleral buckling $\cdot$ Vitrectomy

\section{Introduction}

Retinal detachment with inferior breaks or multiple breaks may be associated with unfavorable success when treated with vitrectomy and gas alone due to reduced support of the

Sabine Baumgarten

sabaumgarten@ukaachen.de

1 Department of Ophthalmology, RWTH Aachen University, Pauwelsstr. 30, 52074 Aachen, Germany

2 Institute of Medical Statistics and Computational Biology, University of Cologne, Cologne, Germany

3 Eye Center, Albert-Ludwigs-University of Freiburg, Freiburg im Breisgau, Germany

4 University Eye Hospital, Hannover Medical School, Hannover, Germany

5 Department of Ophthalmology, University of Regensburg, Regensburg, Germany

6 Department of Ophthalmology, Franziskus Hospital, Münster, Germany inferior breaks by the endotamponade. Accordingly, increased anatomical and functional failure has been reported in such cases [1-3]. The prospective randomized controlled VIPER study showed that an additional encircling band (EB) does not significantly reduce the risk of any second procedure necessary to reattach the retina in patients with primary pseudophakic retinal detachment [4,5]. However, an additional buckle may still be helpful in patients with inferior or multiple breaks. The aim of this post hoc analysis was to test if an additional buckle increases anatomical success compared to $20 \mathrm{G}$ vitrectomy without additional buckle in the subgroup of patients with inferior breaks as well as in the subgroup of patients with multiple breaks (4 or more breaks).

\section{Methods}

The VIPER Study (Vitrectomy with and without encircling band in the treatment of $p$ seudophakic retinal detachment) was a prospective, multicenter, randomized clinical trial registered in the German Register for Clinical Trials (DRKS 00003158, www.germanctr.de). A total of 34 vitreoretinal 
surgeons in 14 clinical sites across Germany participated. The study protocol was approved by each local ethics committee. Details of the study methods and the primary analysis have been described in a design publication [5]. Briefly, 257 patients with uncomplicated pseudophakic retinal detachment were randomized to the following groups:

Control group (C): In this group, patients received a $20 \mathrm{G}$ vitrectomy without encircling band. Surgery started with opening the conjunctiva at the limbus to uncover the sclera. With a distance of 3-4 $\mathrm{mm}$ to the limbus, three 20 $G$ sclerotomies were made, and subsequently, a full vitrectomy was performed. A complete vitreous detachment could be realized if the vitreous was not fully detached. Subretinal fluid was drained through heavy liquids. Retinal breaks and high-risk degenerations were treated with endolaser or transconjunctival/transscleral cryopexy once the retina was fully reattached under air or heavy liquids. After full fluid-air exchange, a gas fill using nonexpandable air/gas mixtures such as SF6, C2F6, or C3F8 completed surgery. The sclerotomies and the conjunctiva were sutured and ocular pressure was monitored within $8 \mathrm{~h}$ after surgery and the day after surgery.

Experimental group 1 (E1): Patients received a 20 G vitrectomy with an encircling band. The conjunctiva was opened circumferentially at the limbus and a 2-4-mm encircling band was positioned underneath the recti muscles and subsequently fixated in all four quadrants onto the equator of the globe. Adequate buckling with no choroidal folds should be achieved at the end of surgery. Following, the $20 \mathrm{G}$ vitrectomy was executed as described above for the control group (C).

Experimental group 2 (E2): In this group, patients received a $23 \mathrm{G}$ or $25 \mathrm{G}$ vitrectomy without encircling band. The conjunctiva was shifted and valved or unvalved transconjunctival trocar systems were inserted tangentially. A full vitrectomy was performed and a full vitreous detachment achieved. Subretinal fluid was drained through the use of heavy liquids and/or air. Retinal breaks and high-risk degenerations were treated with endolaser or transconjunctival/transscleral cryopexy. After full fluid-air exchange, a non-expandable air/gas mixture was inserted. At the end of surgery, trocars were removed and sclerotomies were sutured when leaking.

All groups (C, E1, E2): Surgery was executed under an operating microscope and a wide field viewing system (contact/non-contact). The use of triamcinolone, silicon oil, or internal limiting membrane peeling was not allowed as well as a prophylactic laser treatment.

Dependent on surgical skills of the individual surgeon, patients were randomized either with ratio $1: 1$ between $\mathrm{E} 1$ and $\mathrm{C}$, or with ratios 1:1:1 between $\mathrm{E} 1, \mathrm{C}$, and $\mathrm{E} 2$.
The primary endpoint was defined as no indication for any procedure to reattach the retina during the follow-up of 26 weeks. A missing primary endpoint was counted as failure.

For the post hoc analysis, the information on preoperative breaks recorded by the participating surgeons was verified and updated by an evaluation committee (SB, BM). Five different subgroups were compared: Patients with breaks (1) at the 6:00 position, (2) between 5:00 and 7:00, (3) between 4:00 and 8:00 and (4) in the lower hemisphere that is between 3:00 and 9:00, and (5) patients with 4 or more breaks regardless of the localization.

The analysis of the treatment success in the subgroups was based on all subjects, who were randomized and who received surgery. Patients were analyzed for the treatment group to which they were assigned (intention-to-treat principle).

The comparison of $\mathrm{E} 1$ versus $\mathrm{C}$ regarding the primary endpoint, absence of indication for reattaching procedure, was evaluated using the Cochran-Mantel-Haenszel test stratified by surgeon and the corresponding common odds ratio (OR) [6]. Analyses are essentially descriptive; thus, no correction for multiple testing was applied. Statistical analyses were done with SPSS Statistics software (version 13, IBM Corp., Armonk, NY, USA).

\section{Results}

Thirty-eight out of 257 patients (14.8\%; C, 13; E1, 16; E2, 9) had breaks at the 6:00 position and were included in group 1 . Seventy-two patients (28\%; treatment arm C, 31; E1, 28; E2, 13) were included in group 2 with breaks between $5: 00$ and 7:00, 94 patients $(36.6 \%$;, $37 ; \mathrm{E} 1,39 ; \mathrm{E} 2,18)$ were included in group 3 and had breaks between 4:00 and 8:00, and 100 patients $(38.9 \%$;, $41 ; \mathrm{E} 1,41 ; \mathrm{E} 2,18)$ were included in group 4 with breaks in the lower hemisphere.

Sixty-three patients $(24.5 \%$; C, 22; E1, 29; E2, 12) had 4 or more breaks and were included in group 5 . Table 1 shows as example the baseline characteristics of patients with inferior breaks between 5:00 and 7:00 in the treatment arm $\mathrm{C}$ compared to E1. The preoperative characteristics were fairly balanced among the treatment arms.

Considering all patients regardless of the location of breaks, the primary anatomical success rate was $79 \%$ in the arm treated with $20 \mathrm{G}$ vitrectomy plus an encircling band and $73.5 \%$ in the control arm without encircling band (OR 1.32, CI 0.65 to 2.65; Fig. 1) [7]. If only patients with inferior breaks are considered, the percentage with an anatomical success was higher in the treatment arm with encircling band compared to control (for location of breaks 3-9: 78\% with EB vs. $68.3 \%$ without EB; location of breaks 4-8: $75.7 \%$ vs. $66.7 \%$; Fig. 1). This difference was most distinct in patients with breaks between 5:00 and 7:00 with $77.4 \%$ (24/31, treatment arm E1) versus $57.1 \%(16 / 28$, treatment arm $\mathrm{C})$. The odds ratio (E1 
Table 1 Preoperative characteristics of patients in subgroup with breaks between 5:00 and 7:00

\begin{tabular}{|c|c|c|}
\hline \multirow[t]{2}{*}{ Characteristic } & \multicolumn{2}{|l|}{ Surgery } \\
\hline & $\begin{array}{l}\text { (E1) } 20 \text { G vitrectomy with EB } \\
(n=31) \\
\text { Summary statistics }\end{array}$ & $\begin{array}{l}\text { (C) } 20 \mathrm{G} \text { vitrectomy without } \mathrm{EB} \\
(n=28) \\
\text { Summary statistics }\end{array}$ \\
\hline Sex, male & $23(74.2 \%)$ & $19(67.9 \%)$ \\
\hline Age (years) & $65 \pm 9$ & $66 \pm 10$ \\
\hline Study eye, right & $19(61.3 \%)$ & $11(39.3 \%)$ \\
\hline Sphere (diopter) & $0.25(0.00$ to 1.00$)$ & $0.00(-1.25$ to 0.00$)$ \\
\hline Cylinder (diopter) ${ }^{\ddagger}$ & $-0.50(-1.25$ to 0.00$)$ & $-1.00(-1.00$ to 0.00$)$ \\
\hline Axis (degree) $)^{\ddagger}$ & $40(0$ to 140$)$ & $57(0$ to 110$)$ \\
\hline $\begin{array}{l}\text { Intraocular pressure } \\
\qquad(\mathrm{mmHg})^{\frac{f}{t}}\end{array}$ & 14 (12 to 17$)$ & 15 (12 to 18$)$ \\
\hline Visual acuity, $\log \mathrm{MAR}^{\ddagger}$ & $1.6(0.8$ to 1.7$)$ & $0.7(0.2$ to 1.7$)$ \\
\hline \multicolumn{3}{|c|}{ Vitreous situation at start of surgery } \\
\hline Fully attached & $2(6.5 \%)$ & $2(7.1 \%)$ \\
\hline Partly attached & $9(29.0 \%)$ & $7(25.0 \%)$ \\
\hline Fully detached & $19(61.3 \%)$ & $17(60.7 \%)$ \\
\hline Hemorrhage & $0(0.0 \%)$ & $1(3.6 \%)$ \\
\hline Other & $1(3.2 \%)$ & $1(3.6 \%)$ \\
\hline
\end{tabular}

${ }^{\star}$ Percentage of missing data $\leq 3.6 \%$; otherwise complete data with regard to C) was 1.83 (95\% confidence interval (CI) 0.48 to $7.17, p=0.301)$. However, in none of the comparisons, the odds ratio differed significantly from 1 .

In patients with 4 or more breaks, the respective success rates were $68.2 \%$ (15/22, treatment arm E1) versus $72.4 \%$ (21/ 29 , treatment arm $\mathrm{C}$, odds ratio $0.52,95 \%$ confidence interval 0.08 to $3.65, p=0.46$; Fig. 1 ).

\section{Discussion}

Whereas some authors have reported favorable results in treating retinal detachments with inferior breaks by vitrectomy alone $[8,9]$, others found superior primary attachment rates in their patients treated with vitrectomy plus buckling compared to published results without additional buckle [10]. To our knowledge, the only study comparing the two treatments in retinal detachment with inferior breaks using a control group is a retrospective study by Wickham et al. [11]. The authors reported on 86 patients with retinal detachment and inferior breaks with a primary anatomical success rate of $89 \%$ if treated with vitrectomy and gas and $73 \%$ if treated with vitrectomy and additional buckle without significant difference. Because of the retrospective design of the study, bias in the assignment of the treatment may be assumed. Accordingly, the authors report a higher rate of proliferative vitreoretinopathy in the combined treatment group (20\% vs. $5 \%$ ).

In our investigation, we found that the anatomical success was higher in patients with inferior breaks when treated with an additional encircling band compared to the control group without EB.

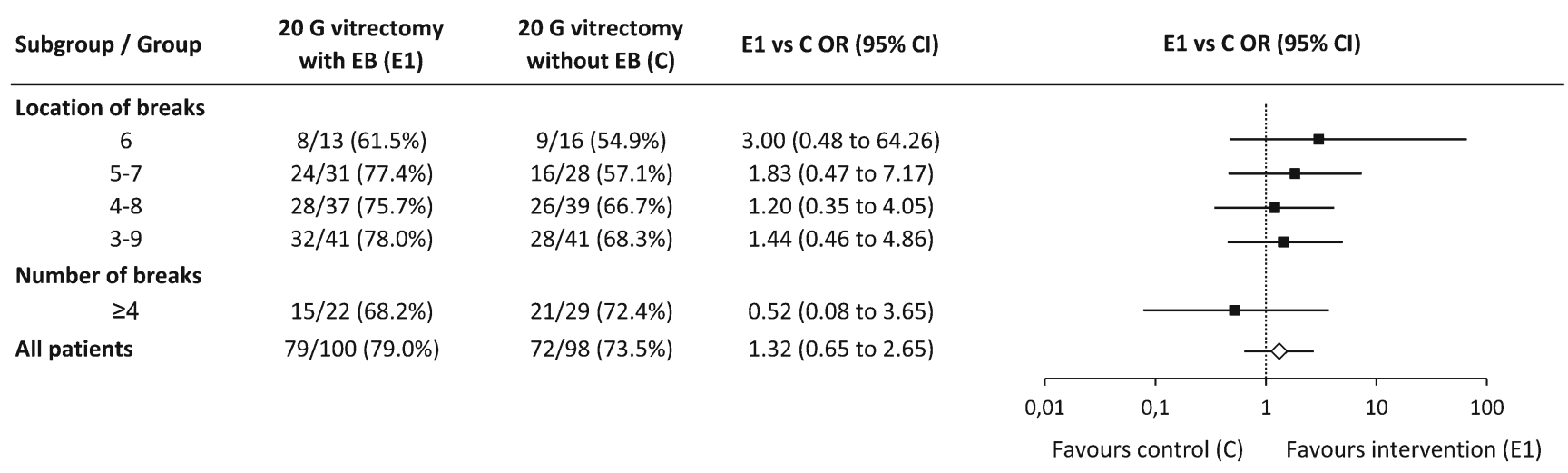

Fig. 1 Primary outcome analysis with respect to the subgroups. (Primary outcome is defined as absence of indication for reattaching procedure; thus, the odds ratio (OR) states the chance of achieving a successful outcome after vitrectomy with EB (E1) compared to vitrectomy without $\mathrm{EB}(\mathrm{C}), \mathrm{CI}$ confidence interval.) 
This trend was most pronounced in group 2 with breaks between 5:00 and 7:00 (77.4\% vs. 57.1\%). The odds ratio of 1.83 indicates an increasing chance of success if an encircling band is added as compared to control, though the results were not significant. By including breaks at more superior locations in the inferior hemisphere, the need for additional buckling may be reduced by a better effect of the endotamponade. When focusing on breaks at the 6:00 position (group 1), where the endotamponade can be assumed least effective and therefore an additional buckle most helpful, only 38 patients could be included in the present analysis which may account for the less distinct results compared to group 2. Our results show a trend that the combined procedure with vitrectomy and encircling band in pseudophakic retinal detachments with inferior breaks might be beneficial. However, improvement of the outcome was not statistically significant. In patients with multiple ( 4 or more) breaks regardless of the position, the success is similar in both treatment groups.

Our study is limited by the relatively small number of included subjects due to focusing on cases with inferior or multiple breaks, which may account for the failure to show a significant effect of additional buckling. The low success rates in any groups are because of the complicate cases of retinal detachments. Having inferior or multiple breaks provides a difficult starting position. In Viper Report No. 2, we reported on our results regardless of the break position. Success rates here were higher (E1, $79 \%$ vs. C, $73.5 \%$ and E2, $87.7 \%$ vs. C, $78.7 \%$ [7]). To the best of our knowledge, this is the only study that excluded bias in treatment assignment due to the randomized controlled study design.

Acknowledgements This work was sponsored by retina.net, a German competence network for clinical studies in retinal diseases. Retina.net provided the communication infrastructure for the VIPER Study Group and the resources for the Internet randomization and statistics as well as the setup and management of the data entry system. More information on retina.net is given under www.retina-net.uni-koeln.de.

The VIPER Study Group consisted of the following investigators and subinvestigators:

P. Walter ${ }^{1}$, B. Mazinani ${ }^{1}$, S. Baumgarten ${ }^{1}$, P. Schiller ${ }^{2}$, M. Hellmich ${ }^{2}$, G. Rössler ${ }^{1}$, H. Agostini ${ }^{3}$, B. Junker ${ }^{4}$, H. Helbig ${ }^{5}$, A. Lommatzsch ${ }^{6}$, F. Holz $^{7}$, B. Kirchhof ${ }^{8}$, E. Limburg 9 , K.-U. Bartz-Schmidt ${ }^{10}$, A. Joussen ${ }^{11}$,

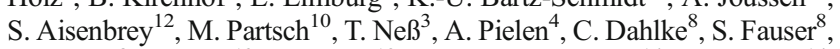
A. Lappa ${ }^{8}$, N. Eter $^{13}$, C. Uhlig ${ }^{13}$, U. Ritzau-Tondrow ${ }^{14}$, N. Feltgen ${ }^{14}$, M.A. Gamulescu ${ }^{5}$, M. Rudolf ${ }^{15}$, M. Lüke ${ }^{15}$, M. Gök ${ }^{16}$, P. Wiedemann $^{17}$, C. Jochmann ${ }^{17}$, P. Meier ${ }^{17}$, A. Nestler ${ }^{17}$, W. Rasche ${ }^{17}$, C. Clemens ${ }^{13}$, J. Hillenkamp ${ }^{18}$, H. Roider ${ }^{19}$

(1) Department of Ophthalmology, RWTH Aachen University; (2) Institute of Medical Statistics and Computational Biology, University of Cologne; (3) Eye Center, Albert-Ludwigs-University of Freiburg; (4) University Eye Hospital, Hannover Medical School, Hannover; (5) Department of Ophthalmology, University of Regensburg; (6) Department of Ophthalmology, Franziskus Hospital, Münster; (7) Department of Ophthalmology, University of Bonn; (8) Center of Ophthalmology, University of Cologne; (9) Center for Clinical Trials, University of Cologne; (10) Centre of Ophthalmology, University of Tübingen; (11) Department of Ophthalmology, Charité Berlin University of Medicine; (12) Department of Ophthalmology, Vivantes Hospital Neukölln, Berlin; (13) Department of Ophthalmology,
University of Münster; (14) Department of Ophthalmology, University of Göttingen; (15) Department of Ophthalmology, University of Schleswig-Holstein, Lübeck; (16) Department of Ophthalmology, University of Essen; (17) Department of Ophthalmology, University of Leipzig; (18) Department of Ophthalmology, University of Würzburg; (19) Department of Ophthalmology, University of Kiel

The authors thank H. Heimann for continuous discussion and support for the design of the VIPER Study.

Funding The study was supported by retina.net, the German Competence Network for Clinical Studies in Vitreoretinal Surgery. Retina.net received grants from the Jackstädt Foundation, the German Retina Society, and the German Ophthalmological Society (DOG). Moreover, ZKS Köln was supported by BMBF grant $01 \mathrm{KN} 1106$. These sponsors had no role in the design or conduct of this research.

\section{Compliance with ethical standards}

Conflict of interest The authors declare that they have no conflict of interest.

Ethical approval All procedures performed in studies involving human participants were in accordance with the ethical standards of the institutional and/or national research committee and with the 1964 Helsinki declaration and its later amendments or comparable ethical standards.

Informed consent Informed consent was obtained from all individual participants included in the study.

Open Access This article is distributed under the terms of the Creative Commons Attribution 4.0 International License (http:// creativecommons.org/licenses/by/4.0/), which permits unrestricted use, distribution, and reproduction in any medium, provided you give appropriate credit to the original author(s) and the source, provide a link to the Creative Commons license, and indicate if changes were made.

\section{References}

1. Goto T, Nakagomi T, Iijima H (2013) A comparison of the anatomic successes of primary vitrectomy for rhegmatogenous retinal detachment with superior and inferior breaks. Acta Ophthalmol 91(6): 552-556. https://doi.org/10.1111/j.1755-3768.2012.02455.x

2. Heussen N, Feltgen N, Walter P, Hoerauf H, Hilgers RD, Heimann H (2011) Scleral buckling versus primary vitrectomy in rhegmatogenous retinal detachment study (SPR Study): predictive factors for functional outcome. Study report no. 6. Graefes Arch Clin Exp Ophthalmol 249(8):1129-1136. https://doi.org/10.1007/ s00417-011-1619-7

3. Heimann H, Zou X, Jandeck C, Kellner U, Bechrakis NE, Kreusel KM, Helbig H, Krause L, Schuler A, Bornfeld N, Foerster MH (2006) Primary vitrectomy for rhegmatogenous retinal detachment: an analysis of 512 cases. Graefes Arch Clin Exp Ophthalmol 244(1):69-78. https://doi.org/10.1007/s00417-005-0026-3

4. Walter P, Hellmich M, Baumgarten S, Schiller P, Limburg E, Agostini H, Pielen A, Helbig H, Lommatzsch A, Rossler G, Mazinani B, Group VS (2016) Vitrectomy with and without encircling band for pseudophakic retinal detachment: VIPER Study Report No 2-main results. Br J Ophthalmol. https://doi.org/ 10.1136/bjophthalmol-2016-309240

5. Mazinani B, Baumgarten S, Schiller P, Agostini H, Helbig H, Limburg E, Hellmich M, Walter P, Group VS (2016) Vitrectomy with 
or without encircling band for pseudophakic retinal detachment: a multi-centre, three-arm, randomised clinical trial. VIPER Study Report No. 1-design and enrolment. Br J Ophthalmol 100(3):405410. https://doi.org/10.1136/bjophthalmol-2015-306732

6. Mantel N, Haenszel W (1959) Statistical aspects of the analysis of data from retrospective studies of disease. J Natl Cancer Inst 22(4):719-748

7. Walter P, Hellmich M, Baumgarten S, Schiller P, Limburg E, Agostini H, Pielen A, Helbig H, Lommatzsch A, Rossler G, Mazinani B (2017) Vitrectomy with and without encircling band for pseudophakic retinal detachment: VIPER Study Report No 2main results. Br J Ophthalmol 101(6):712-718. https://doi.org/10. 1136/bjophthalmol-2016-309240

8. Sharma A, Grigoropoulos V, Williamson TH (2004) Management of primary rhegmatogenous retinal detachment with inferior breaks.
Br J Ophthalmol 88(11):1372-1375. https://doi.org/10.1136/bjo. 2003.041350

9. Tanner V, Minihan M, Williamson TH (2001) Management of inferior retinal breaks during pars plana vitrectomy for retinal detachment. Br J Ophthalmol 85(4):480-482

10. Alexander P, Ang A, Poulson A, Snead MP (2008) Scleral buckling combined with vitrectomy for the management of rhegmatogenous retinal detachment associated with inferior retinal breaks. Eye (Lond) 22(2):200-203. https://doi.org/10.1038/sj.eye.6702555

11. Wickham L, Connor M, Aylward GW (2004) Vitrectomy and gas for inferior break retinal detachments: are the results comparable to vitrectomy, gas, and scleral buckle? Br J Ophthalmol 88(11):13761379. https://doi.org/10.1136/bjo.2004.043687 